\title{
Thiazolidinediones: Do harms outweigh benefits?
}

\author{
Lorraine L. Lipscombe MD MSc
}

Published at www.cmaj.ca on Dec. 10, 2008.

$\infty \infty$

See related research paper by Loke and colleagues, page 32

$\mathrm{T}$ here has been recent debate about the risks and benefits of the use of thiazolidinediones (rosiglitazone and pioglitazone) to treat diabetes. Similar to all other drugs used to treat diabetes, thiazolidinediones were approved by drug regulatory bodies based on their ability to improve glycemic control, rather than based on long-term clinical benefit. However, recent evidence suggests that these drugs may be associated with an increased risk of cardiovascular events and fractures, leading to heightened controversy regarding the appropriate role of thiazolidinediones in the treatment of diabetes. In this issue of CMAJ, Loke and colleagues ${ }^{1}$ report the findings of a meta-analysis of the impact of thiazolidinediones on risk of fracture and bone loss among patients with diabetes.

Based on 10 randomized controlled trials that lasted at least 12 months and involved over 13000 patients, Loke and colleagues found that thiazolidinediones were associated with a $45 \%$ increase in fractures. Consistent with previous reports, the risk appeared to be limited to women. Similar results were found when data from 2 observational studies were pooled. Use of thiazolidinedione was also associated with significant bone loss. The authors estimate that for every 55 women at low-risk of fracture who take a thiazolidinedione for 1 year, 1 fracture would occur. Among women at high risk, 1 fracture would occur for every 21 women who take thiazolidinedione for 1 year.

When thiazolidinediones were first introduced, they held great promise for reduction of cardiovascular risk among people with type 2 diabetes mellitus. In addition to improved glycemic control without hypoglycemia, thiazolidinediones decrease insulin resistance, a key risk factor for cardiovascular disease. ${ }^{2}$ These features, along with evidence of benefit on surrogate cardiovascular markers, rapidly led to the widespread use of these drugs.

Concerns about thiazolidinediones began to emerge shortly after their introduction. Troglitazone, the first thiazolidinedione introduced, was withdrawn from the market in 2000 because of rare cases of severe hepatotoxicity. Rosiglitazone and pioglitazone were approved for use in 1999. Although there was less hepatotoxicity, these drugs were associated with weight gain, edema and increased risk of heart failure. ${ }^{3}$ In 2007, a meta-analysis based on 42 randomized trials, which was later criticized because of methodologic concerns, suggested that rosiglitazone increased the risk of myocardial infarction and cardiovascular death. ${ }^{4}$ Two other meta-

\section{Key points \\ - Thiazolidinediones may be associated with a higher risk of fracture in women with type 2 diabetes mellitus. \\ - The risks and benefits of thiazolidinedione therapy should be carefully considered. \\ - Until further evidence of their net benefit is available, the appropriate role for these drugs is unclear. \\ - Clinical drug trials are often underpowered to detect unanticipated and rare adverse effects, and a standardized postmarketing surveillance process is needed.}

analyses, ${ }^{5,6}$ including one from the US Food and Drug Administration (FDA), and a large observational study ${ }^{7}$ reported similar findings. These findings prompted the FDA to add a black-box warning to the drug label about potential severe adverse outcomes and prompted Health Canada to place new restrictions on the use of thiazolidinediones. These publications received widespread media attention and caused considerable public alarm.

These findings lead us to question the basis on which drugs to treat diabetes are approved. Traditionally, regulatory bodies such as Health Canada and the FDA have approved medications for diabetes based on short-term outcomes such as glycemic control. This approach may be shortsighted, especially given that glycemic control has not been convincingly shown to reduce cardiovascular events, which is the most important source of morbidity and mortality among patients with type 2 diabetes mellitus. The recent evidence that thiazolidinediones may instead achieve the opposite results underscores the need to show benefits on clinical outcomes rather than surrogate markers.

However, even if long-term clinical outcomes are assessed, it is often difficult to detect unexpected adverse effects not linked to clinical benefit. This is especially true for less common events, because clinical trials are often underpowered to detect infrequent adverse effects of drugs. Thus, in the study by Loke and colleagues, the lack of an observed effect of thiazolidinediones among men as well as on hip fractures may simply be because of insufficient power. Also, because patients in trials are usually healthier than patients in regular

Dr. Lipscombe is with the Institute for Clinical Evaluative Sciences, the Department of Medicine, University of Toronto, and Women's College Hospital, Toronto, Ont. 
clinical settings, trial participants may be at lower baseline risk of adverse outcomes. Thus, reliance on clinical trials may miss important risks associated with new drugs and underestimate the ratio of risks to benefits in populations at high risk for adverse events. These risks are often unanticipated despite a good understanding of the drug's mechanisms.

All drugs have potential side effects that need to be weighed against the potential benefits. Given the growing evidence of harms, do the benefits of thiazolidinedione therapy still outweigh the risks? These drugs may improve glycemic control for patients who have achieved inadequate glycemic control with other hypoglycemic agents, particularly if insulin therapy is a less feasible option. Moreover, there may be differences between rosiglitazone and pioglitazone with respect to cardiovascular risk. Regardless, both drugs are associated with a higher risk of heart failure and fracture. Therefore, the net benefit of thiazolidinedione therapy is unclear. Given that there are other effective drugs to control glycemia that are associated with fewer adverse events, thiazolidinediones should not be considered appropriate as first-line therapy for type 2 diabetes mellitus. If a patient is unable to take other therapies or if other therapies have failed, there may be a role for thiazolidinediones in carefully selected patients duly informed of the potential adverse effects. Considering that studies of pioglitazone have not shown the possible higher risk of myocardial infarction seen with rosiglitazone, but rather suggest a reduction in ischemic events,, 89 pioglitazone may be a safer option.

The ongoing accumulation of evidence of harm for thiazolidinediones is unsettling to clinicians and patients and threatens to undermine patient confidence. The study by Loke and colleagues highlights the need for routine and standardized postmarketing surveillance strategies to be implemented for new drugs, so that both patients and clinicians can be assured that unexpected adverse effects are monitored and reported on a regular basis. The need for new therapies must be balanced with more careful drug approval and pharmacovigilance processes, so that the safety of patients can be enhanced.

This article has been peer reviewed.

Competing interests: None declared.

Acknowledgements: I thank Dr. Janet Hux and Dr. Rodrigo Cavalcanti for their thoughtful comments and feedback.

Lorraine Lipscombe is the recipient of a Clinician-Scientist Award from the Canadian Diabetes Association and the Canadian Institutes for Health Research.

\section{REFERENCES}

1. Loke YK, Singh S, Furberg C. Long-term use of thiazolidinediones and fractures in type 2 diabetes: a meta-analysis. CMAJ 2009;180(1):32-9.

2. Yki-Jarvinen H. Thiazolidinediones. N Engl J Med 2004;351:1106-18.

3. Lago RM, Singh PP, Nesto RW. Congestive heart failure and cardiovascular death in patients with prediabetes and type 2 diabetes given thiazolidinediones: a metaanalysis of randomised clinical trials. Lancet 2007;370:1129-36.
4. Nissen SE, Wolski K. Effect of rosiglitazone on the risk of myocardial infarction and death from cardiovascular causes. N Engl J Med 2007;356:2457-71.

5. Singh S, Loke YK, Furberg CD. Long-term risk of cardiovascular events with rosiglitazone: a meta-analysis. JAMA 2007;298:1189-95.

6. United States Food and Drug Administration. FDA briefing document: joint meeting of the Endocrinologic and Metabolic Drug Advisory Committee and the Drug Safety and Risk Management Committee. Available: www.fda.gov/ohrms/dockets /ac/07/briefing/2007-4308b1-00-index.htm (accessed 2008 Nov 4).

7. Lipscombe LL, Gomes T, Levesque LE, et al. Thiazolidinediones and cardiovascular outcomes in older patients with diabetes. JAMA 2007;298:2634-43.

8. Dormandy JA, Charbonnel B, Eckland DJ, et al. Secondary prevention of macrovascular events in patients with type 2 diabetes in the PROactive Study (PROspective pioglitAzone Clinical Trial In macroVascular Events): a randomised controlled trial. Lancet 2005;366:1279-89.

9. Lincoff AM, Wolski K, Nicholls SJ, et al. Pioglitazone and risk of cardiovascular events in patients with type 2 diabetes mellitus: a meta-analysis of randomized trials. JAMA 2007;298:1180-8.

Correspondence to: Dr. Lorraine Lipscombe, Institute for Clinical Evaluative Sciences, 2075 Bayview Ave., Toronto ON M4N 3M5; fax 416 323-6534; Lorraine.Lipscombe@ices.on.ca

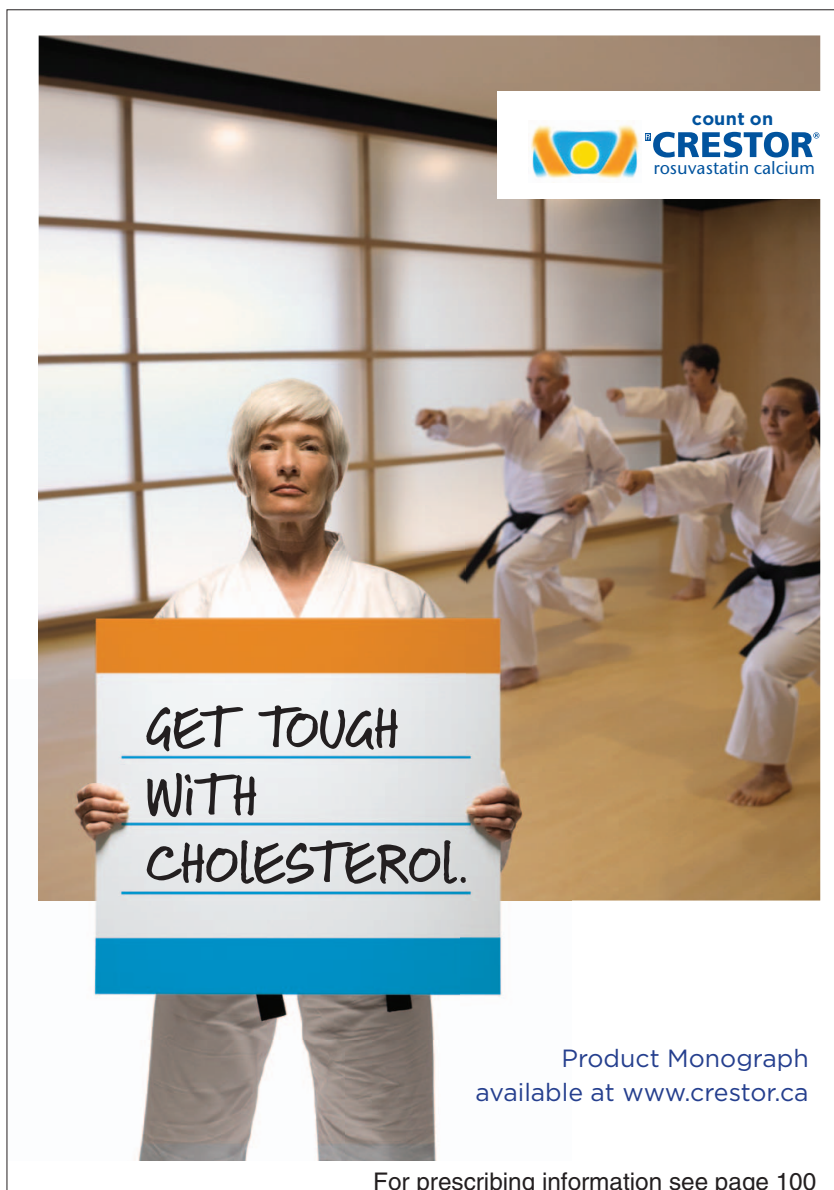

For prescribing information see page 100 